Pacific

Journal of

Mathematics

PARAHORIC FIXED SPACES IN UNRAMIFIED PRINCIPAL SERIES REPRESENTATIONS

JOSHUA M. LANSKY 


\title{
PARAHORIC FIXED SPACES IN UNRAMIFIED PRINCIPAL SERIES REPRESENTATIONS
}

\author{
JOSHUA M. LANSKY
}

Let $k$ be a non-archimedean locally compact field and let $G$ be the set of $k$-points of a connected reductive group defined over $k$. Let $W$ be the relative Weyl group of $G$, and let $\mathcal{H}(G, B)$ be the Hecke algebra of $G$ with respect to an Iwahori subgroup $B$ of $G$. We compute the effects of $\mathcal{H}(G, B)$ and $W$ on the $B$-fixed vectors of an unramified principal series representation $I$ of $G$. We use this computation to determine the dimension of the space of $K$-fixed vectors in $I$, where $K$ is a parahoric subgroup of $G$.

\section{Introduction.}

Let $\mathbf{G}$ be a reductive group defined over a non-archimedean locally compact field $k$ and let $G=\mathbf{G}(k)$. Let $P$ be a minimal parabolic subgroup of $G$ with Levi decomposition $P=M N$, and let $P^{-}=M N^{-}$be the corresponding decomposition of the opposite parabolic $P^{-}$. Let $B$ be an Iwahori subgroup of $G$ with an Iwahori decomposition with respect to $P$ and $M$, i.e.,

$$
B=(B \cap P)(B \cap M)\left(B \cap P^{-}\right) .
$$

Denote by $W$ the relative Weyl group of $G$. Let $\chi$ be an unramified character of $M$ (i.e., $\chi$ is trivial on $M_{0}$ ). Since $M \cong P / N, \chi$ extends to a character of $P$ which we will also denote by $\chi$. Let $\delta$ be the modulus character of $P$. Define $I(\chi)$ to be the unramified principal series representation of $G$ induced by $\chi$, i.e., the space of all locally constant functions $G \rightarrow \mathbb{C}$ such that

$$
f(p g)=\chi \delta^{1 / 2}(p) f(g) \text { for all } p \text { in } P, g \text { in } G
$$

on which $G$ acts by right translation. It is well-known that the space $I(\chi)^{B}$ of $B$-fixed vectors in $I(\chi)$ has dimension $\operatorname{dim} I(\chi)^{B}=|W|$ [3, Prop. 2.1]. In this paper, we generalize this result to the fixed space $I(\chi)^{K}$ where $K$ is a parahoric subgroup of $G$ containing $B$.

Let $A$ be a maximal split torus in $M$ and let $\mathcal{N}$ be its normalizer in $G$. If $M_{0}$ is the maximal compact subgroup of $M$ and $\widetilde{W}=\mathcal{N} / M_{0}$, then we have a surjection $\nu: \widetilde{W} \rightarrow W=\mathcal{N} / M$. Let $K$ be a parahoric subgroup of $G$ containing $B$ and let $W_{K}$ be the finite Coxeter subgroup of $\widetilde{W}$ such that $K=B W_{K} B$ (see $\left.[4, \S 1]\right)$. We will prove the following: 
Theorem 1.1. The dimension of $I(\chi)^{K}$ is $\left|W / \nu\left(W_{K}\right)\right|$.

As a Coxeter group, $W_{K}$ is generated by a canonical finite set $S$ of reflections. Thus

$$
I(\chi)^{K}=\bigcap_{s \in S} I(\chi)^{\langle B, s\rangle} .
$$

In Section 3, we explicitly determine the effects of reflections $s \in S$ on $I(\chi)^{B}$ (Theorem 3.1) and as a corollary the actions of the generators of the Iwahori-Hecke algebra $\mathcal{H}(G, B)$ on $I(\chi)^{B}$ (Corollary 3.2). We then compute the subspaces $I(\chi)^{\langle B, s\rangle}$ in terms of the usual basis of $I(\chi)$ as given in [3, Prop. 2.1]. Then in Section 4, we complete the proof of Theorem 1.1 by showing that the dimension of the intersection of the $I(\chi)^{\langle B, s\rangle}$ is $\left|W / \nu\left(W_{K}\right)\right|$.

Let $\mathcal{H}(G, K)$ be the Hecke algebra of compactly supported functions $G \rightarrow$ $\mathbb{C}$, bi-invariant by $K$. Let $E$ be a simple $\mathcal{H}(G, K)$-module. It is known that there is an irreducible admissible representation $V$ of $G$ such that $E$ is isomorphic as a $\mathcal{H}(G, K)$-module to the space $V^{K}$ of $K$-fixed vectors [1, 2.10]. Since $V^{B} \supset V^{K}=E \neq 0$, it follows from a well-known result that $V$ embeds inside some unramified principal series representation $I$ of $G$ so that $\operatorname{dim} E=\operatorname{dim} V^{K} \leq \operatorname{dim} I^{K}$. Thus Theorem 1.1 has the following corollary:

Corollary 1.2. If $K$ is a parahoric subgroup of $G$ and $E$ is a simple module over $\mathcal{H}(G, K)$, then

$$
\operatorname{dim} E \leq\left|W / \nu\left(W_{K}\right)\right| .
$$

Moreover, this bound is sharp.

The sharpness of this bound is a result of the fact that there exist irreducible unramified principal series representations (see e.g., [2, Theorem $3.3])$ and that for such a representation $I$, the $\mathcal{H}(G, K)$-module $I^{K}$ is simple $[1,2.10]$ and, by Theorem 1.1 , of dimension $\left|W / \nu\left(W_{K}\right)\right|$.

Remark 1.3. While Theorem 1.1 is needed to prove the sharpness in Corollary 1.2, the inequality itself can be proved by a simpler argument. Indeed, it is easily demonstrated that $\operatorname{dim} I(\chi)^{K} \leq\left|W / \nu\left(W_{K}\right)\right|$ by noting that

$$
\operatorname{dim} I(\chi)^{K} \leq|P \backslash G / K|
$$

and

$$
|P \backslash G / K|=\left|W / \nu\left(W_{K}\right)\right| .
$$

I would like to express my gratitude to both Benedict Gross and David Pollack for their many helpful suggestions for this paper.

\section{Preliminaries.}

See $[\mathbf{6}]$ or $[\mathbf{3}, \S 1]$ as a reference for much of the material in this section. In the following, we let $k$ be a non-archimedean locally compact field. We denote by $\mathbf{G}$ a connected reductive algebraic group defined over $k$ with group of 
$k$-points $G$. Similarly, throughout this section, if $\mathbf{H}$ is any algebraic group defined over $k$, we will denote its $k$-points by the corresponding non-bold letter $H$.

Let $\mathbf{P}$ be a fixed minimal parabolic subgroup of $\mathbf{G}$ containing a maximal split torus $\mathbf{A}$ of $\mathbf{G}$. Denote by $\mathbf{N}$ the unipotent radical of $\mathbf{P}$, and by $\mathbf{M}$ the centralizer of $\mathbf{A}$. Then $\mathbf{P}$ has Levi decomposition $\mathbf{M N}$. Let $\Phi^{\prime}$ denote the set of roots of $\mathbf{G}$ relative to $\mathbf{A}$ and $\Phi_{\text {nd }}^{\prime}$ the subset of non-divisible roots. Also, let $W$ be the relative Weyl group.

Denote by $\mathcal{B}=\mathcal{B}(\mathbf{G}, k)$ the Bruhat-Tits building of $\mathbf{G}$ over $k$ and by $\mathcal{A}$ the apartment of $\mathcal{B}$ stabilized by $A$. The normalizer $\mathcal{N}$ of $A$ in $G$ is then the stabilizer of $\mathcal{A}$ and the maximal compact subgroup $M_{0}$ of $M$ is the kernel of the map $\mathcal{N} \rightarrow \operatorname{Aut}(\mathcal{A})$. Let $\widetilde{W}=\mathcal{N} / M_{0}$. Denote by $\Phi_{\text {aff }}$ the canonical affine root system on $\mathcal{A}$ and by $W_{\text {aff }}$ the corresponding affine Weyl group. Then $W_{\text {aff }}$ may be identified with a normal subgroup of $\widetilde{W}$.

Fix a special point $x_{0}$ in $\mathcal{B}$ and let $\Phi$ be the set of affine roots vanishing at $x_{0}$. Then $\Phi$ is a reduced root system, and we have a bijection between $\Phi$ and $\Phi_{\text {nd }}^{\prime}$ corresponding to the choice of $x_{0}$. We let $\Phi^{+}$be the subset of positive affine roots corresponding to $P$ and $\Delta$ the subset of simple roots.

Let $C$ be the unique chamber in $\mathcal{A}$ containing $x_{0}$ with the property that every $\alpha$ in $\Phi^{+}$takes positive values on $C$. Denote by $B$ the Iwahori subgroup of $G$ fixing $C$ pointwise and by $K_{0}$ the special maximal compact subgroup fixing $x_{0}$. Then $W=\mathcal{N} / M \cong\left(\mathcal{N} \cap K_{0}\right) / M_{0}$, which is the stabilizer of $x_{0}$ in $\widetilde{W}$. We will identify these groups throughout. We denote by $\nu$ the surjection $\widetilde{W} \rightarrow W$. The kernel of $\nu$ is the group of translations in $\widetilde{W}$.

For each $\alpha$ in $\Phi_{\text {aff }}$, denote by $N(\alpha)$ the pointwise stabilizer of the halfapartment $\{x \in \mathcal{A} \mid \alpha(x) \geq 0\}$. We note that

$$
B=M_{0} \cdot \prod_{\alpha \in \Phi^{+}} N(\alpha) \cdot \prod_{\alpha \in \Phi^{-}} N(\alpha+1) .
$$

Let $P_{0} \subset P$ be the compact subgroup

$$
P \cap K_{0}=M_{0} \cdot \prod_{\alpha \in \Phi^{+}} N(\alpha) .
$$

Let $\Phi=\bigcup \Phi_{i}$ be the decomposition of $\Phi$ into irreducible root systems. Denote by $\widetilde{\Delta}$ the set containing the highest root $\widetilde{\alpha}_{i}$ of $\Phi_{i}$ for each $i$. Let

$$
\Delta_{\text {aff }}=\left\{\alpha \in \Phi_{\text {aff }} \mid \alpha \in \Delta \text { or } \alpha=\widetilde{\alpha}-1 \text { for some } \widetilde{\alpha} \in \widetilde{\Delta}\right\} .
$$

For $\alpha$ in $\Delta_{\text {aff }}$, let $w_{\alpha}$ be the reflection in $\operatorname{Aut}(\mathcal{A})$ through the vanishing hyperplane of $\alpha$. Then $S_{\text {aff }}=\left\{w_{\alpha} \mid \alpha \in \Delta_{\text {aff }}\right\}$ is a set of involutive generators for the Coxeter group $W_{\text {aff }}$.

For $\alpha$ in $\Phi$, let $a_{\alpha}$ be the translation $w_{\alpha} w_{\alpha-1}$ on $\mathcal{A}$. We note that

$$
a_{-\alpha}=a_{\alpha}^{-1} \text { for any } \alpha \text { in } \Phi .
$$


We let $K$ be a fixed parahoric subgroup of $G$ containing $B$. Since the triple $(G, B, \mathcal{N})$ is a generalized Tits system (see $[4, \S 1]$ ), there exists a special subgroup $W_{K}$ of $W_{\text {aff }}$ such that $K=B W_{K} B ; W_{K}$ is finite as $K$ is compact. We denote by $S$ the subset of $S_{\text {aff generating }} W_{K}$.

For any $w$ in $\widetilde{W}$, we denote by $q(w)$ the index $[B w B: B]$. Also for $\alpha$ in $\Phi_{\text {aff }}$, we let $q_{\alpha}$ be the index $[N(\alpha-1): N(\alpha)]$. We note that $q_{\alpha+2}=q_{\alpha}$. Since (cf. [5, Cor. 2.7])

$$
\begin{gathered}
B w_{\alpha} B=N(\alpha) w_{\alpha} B \text { for } \alpha \text { in } \Delta, \\
B w_{\widetilde{\alpha}-1} B=N(-\widetilde{\alpha}+1) w_{\widetilde{\alpha}-1} B \text { for } \widetilde{\alpha} \text { in } \widetilde{\Delta},
\end{gathered}
$$

it follows that

$$
q\left(w_{\alpha}\right)=q_{\alpha+1} \text { for } \alpha \text { in } \Delta, \quad q\left(w_{\widetilde{\alpha}-1}\right)=q_{\widetilde{\alpha}+2}=q_{\widetilde{\alpha}} \text { for } \widetilde{\alpha} \text { in } \widetilde{\Delta} .
$$

If $\alpha \in \Delta$, we denote by $B_{\alpha}$ the group $B \cap w_{\alpha} B w_{\alpha}$, and if $\widetilde{\alpha} \in \widetilde{\Delta}, B_{\widetilde{\alpha}-1}$ denotes the group $B \cap w_{\widetilde{\alpha}-1} B w_{\widetilde{\alpha}-1}$.

Let $d x$ be the Haar measure on $G$ for which $B$ has volume 1 . We denote by $\mathcal{H}(G, B)$ the Iwahori-Hecke algebra of compactly supported functions $G \rightarrow \mathbb{C}$ bi-invariant by $B$. The product on $\mathcal{H}(G, B)$ is given by convolution with respect to $d x$. Fix an unramified character $\chi$ of $M$ and let $\delta$ be the modulus character of $P$. Denote by $I(\chi)$ the induced representation $\operatorname{Ind}_{P}^{G}\left(\chi \delta^{1 / 2}\right)$, i.e., the unramified principal series representation induced by $\chi$ as described in Section 1. If $x$ is an element of $G$, we will denote the action of $x$ on $u \in I(\chi)$ by $u \mapsto x \cdot u$. Note that if $w \in \widetilde{W}$ then the expression $w \cdot u$ is well-defined for $u \in I(\chi)^{B}$ as $w$ is determined modulo $M_{0} \subset B$. A function $h \in \mathcal{H}(G, B)$ acts on $I(\chi)^{B}$ by the formula

$$
h \cdot u=\int_{G}(x \cdot u) h(x) d x
$$

where $v \in I(\chi)^{B}$.

Let $C_{c}^{\infty}(G)$ be the space of locally constant, compactly supported functions $G \rightarrow \mathbb{C}$. The map $\mathcal{P}_{\chi}: C_{c}^{\infty}(G) \rightarrow I(\chi)$ defined by

$$
\mathcal{P}_{\chi}(f)(g)=\int_{P} \chi^{-1} \delta^{1 / 2}(p) f(p g) d p
$$

(where $d p$ is the left Haar measure on $P$ giving $P_{0}$ measure 1 ) is a $G$ equivariant surjection. The functions $\phi_{w, \chi}=\mathcal{P}_{\chi}\left(\operatorname{ch}_{B w B}\right)(w$ in $W)$ form a basis of the subspace of $B$-fixed vectors $I(\chi)^{B}$ [3, Prop. 2.1]. Concretely, for $p \in P, w^{\prime} \in W$ and $b \in B, \phi_{w, \chi}\left(p w^{\prime} b\right)$ equals $\chi \delta^{1 / 2}(p)$ if $w^{\prime}=w$ and is zero otherwise. 


\section{The effect of $W_{\text {aff }}$ on $I(\chi)^{B}$.}

The goal of this section is to compute the effect of $s \in S_{\text {aff }}$ on $I(\chi)^{B}$. This will be important for the proof in the following section since we will need to determine the space $I(\chi)^{\langle B, s\rangle}$ of vectors in $I(\chi)^{B}$ fixed by $s$.

Theorem 3.1. Suppose that $w \in W, \alpha \in \Delta$ and $\widetilde{\alpha} \in \widetilde{\Delta}$. Then

$$
\begin{gathered}
w_{\alpha} \cdot \phi_{w, \chi}= \begin{cases}\operatorname{ch}_{P w w_{\alpha}} B_{\alpha} \phi_{w w_{\alpha}, \chi} & \text { if } w \alpha \in \Phi^{+} \\
\phi_{w w_{\alpha}, \chi}+\operatorname{ch}_{P w\left(B-B_{\alpha}\right)} \phi_{w, \chi} & \text { if } w \alpha \in \Phi^{-},\end{cases} \\
w_{\widetilde{\alpha}-1} \cdot \phi_{w, \chi}= \begin{cases}\chi \delta^{1 / 2}\left(a_{w \widetilde{\alpha}}\right) \operatorname{ch}_{P w w_{\tilde{\alpha}} B_{\widetilde{\alpha}-1}} \phi_{w w_{\tilde{\alpha}}, \chi} & \text { if } w \widetilde{\alpha} \in \Phi^{-} \\
\chi \delta^{1 / 2}\left(a_{w \widetilde{\alpha}}\right) \phi_{w w_{\tilde{\alpha}}, \chi}+\operatorname{ch}_{P w\left(B-B_{\widetilde{\alpha}-1}\right)} \phi_{w, \chi} & \text { if } w \widetilde{\alpha} \in \Phi^{+} .\end{cases}
\end{gathered}
$$

Proof. For any $s$ in $S_{\text {aff }}, g \in G$,

$$
\left(s \cdot \phi_{w, \chi}\right)(g)=\phi_{w, \chi}(g s) .
$$

The Iwasawa decomposition enables us to write $g=p^{\prime} w^{\prime} b^{\prime}$ for some $p^{\prime}$ in $P, w^{\prime}$ in $W$, and $b^{\prime}$ in $B$. We will evaluate $\phi_{w, \chi}(g s)=\phi_{w, \chi}\left(p^{\prime} w^{\prime} b^{\prime} s\right)$ by determining the double coset in which $p^{\prime} w^{\prime} b^{\prime} s$ lies.

We first consider $s=w_{\alpha}$ for $\alpha \in \Delta$. Now if $w^{\prime} \alpha \in \Phi^{+}$then by (1)

$$
\begin{aligned}
p^{\prime} w^{\prime} b^{\prime} w_{\alpha} & \in p^{\prime} w^{\prime} B w_{\alpha} B \\
& =p^{\prime} w^{\prime} N(\alpha) w_{\alpha} B \\
& =p^{\prime} N\left(w^{\prime} \alpha\right) w^{\prime} w_{\alpha} B \\
& \subset\left(p^{\prime} N\right) w^{\prime} w_{\alpha} B .
\end{aligned}
$$

Since $\chi \delta^{1 / 2}$ is trivial on $N$, it follows that $\phi_{w, \chi}\left(p^{\prime} w^{\prime} b^{\prime} w_{\alpha}\right)$ equals $\chi \delta^{1 / 2}\left(p^{\prime}\right)$ if $w=w^{\prime} w_{\alpha}$ and 0 otherwise.

If, on the other hand, $w^{\prime} \alpha \in \Phi^{-}$then suppose first that $b^{\prime} \in B_{\alpha}$. Then

$$
p^{\prime} w^{\prime} b^{\prime} w_{\alpha} \in p^{\prime} w^{\prime} b^{\prime} w_{\alpha} B=p^{\prime} w^{\prime} w_{\alpha} B
$$

since $w_{\alpha} B_{\alpha} w_{\alpha} \subset B$. Thus $\phi_{w, \chi}\left(p^{\prime} w^{\prime} b^{\prime} w_{\alpha}\right)$ equals $\chi \delta^{1 / 2}\left(p^{\prime}\right)$ if $w=w^{\prime} w_{\alpha}$ and 0 otherwise.

Lastly, suppose that $w^{\prime} \alpha \in \Phi^{-}$and $b^{\prime} \in B-B_{\alpha}$. It is easily deduced from $w^{\prime} \alpha \in \Phi^{-}$that

$$
P w^{\prime} B w_{\alpha} B=P w^{\prime} w_{\alpha} B \cup P w^{\prime} B .
$$

Moreover, one can show that $p^{\prime} w^{\prime} b^{\prime} w_{\alpha} \in P w^{\prime} B$ if and only if $b^{\prime}$ is an element of $B-B_{\alpha}$. Thus $p^{\prime} w^{\prime} b^{\prime} w_{\alpha}=p w^{\prime} b$ for some $p \in P, b \in B$. Since

$$
p^{-1} p^{\prime}=w^{\prime} b w_{\alpha} b^{\prime-1} w^{\prime-1} \in P \cap K_{0}=P_{0}
$$

and since $\chi \delta^{1 / 2}$ is trivial on $P_{0}$, we have that $\chi \delta^{1 / 2}(p)=\chi \delta^{1 / 2}\left(p^{\prime}\right)$. Therefore, $\phi_{w, \chi}\left(p^{\prime} w^{\prime} b^{\prime} w_{\alpha}\right)$ equals $\chi \delta^{1 / 2}\left(p^{\prime}\right)$ if $w=w^{\prime}$ and 0 otherwise. 
Note that $w^{\prime} \alpha \in \Phi^{ \pm}$if and only if $w^{\prime} w_{\alpha} \alpha=-w^{\prime} \alpha \in \Phi^{\mp}$. Using this, we assemble the preceding cases to obtain that

$$
\left(w_{\alpha} \cdot \phi_{w, \chi}\right)\left(p^{\prime} w^{\prime} b^{\prime}\right)= \begin{cases}\chi \delta^{1 / 2}\left(p^{\prime}\right) & \text { if } w \alpha \in \Phi^{+}, w^{\prime}=w w_{\alpha}, b^{\prime} \in B_{\alpha} \\ \chi \delta^{1 / 2}\left(p^{\prime}\right) & \text { if } w \alpha \in \Phi^{-}, w^{\prime}=w w_{\alpha} \\ \chi \delta^{1 / 2}\left(p^{\prime}\right) & \text { if } w \alpha \in \Phi^{-}, w^{\prime}=w, b^{\prime} \in B-B_{\alpha} \\ 0 & \text { otherwise }\end{cases}
$$

This immediately implies the first result of the theorem.

We now prove the second formula by calculating $w_{\widetilde{\alpha}-1} \cdot \phi_{w, \chi}$ for $\widetilde{\alpha} \in \widetilde{\Delta}$. Assume first that $w^{\prime} \widetilde{\alpha} \in \Phi^{-}$. Then by (2)

$$
\begin{aligned}
p^{\prime} w^{\prime} b^{\prime} w_{\widetilde{\alpha}-1} & \in p^{\prime} w^{\prime} B w_{\widetilde{\alpha}-1} B \\
& =p^{\prime} w^{\prime} N(-\widetilde{\alpha}+1) w_{\widetilde{\alpha}-1} B \\
& =p^{\prime} N\left(-w^{\prime} \widetilde{\alpha}+1\right) w^{\prime} w_{\widetilde{\alpha}} a_{\widetilde{\alpha}} B \\
& \subset\left(p^{\prime} a_{-w^{\prime} \widetilde{\alpha}} N\right) w^{\prime} w_{\widetilde{\alpha}} B .
\end{aligned}
$$

Since $\chi$ is trivial on $N$, it follows that $\phi_{w, \chi}\left(p^{\prime} w^{\prime} b^{\prime} w_{\widetilde{\alpha}-1}\right)$ equals $\chi \delta^{1 / 2}\left(p^{\prime} a_{-w^{\prime} \widetilde{\alpha}}\right)$ if $w=w^{\prime} w_{\widetilde{\alpha}}$ and 0 otherwise.

Now suppose that $w^{\prime} \widetilde{\alpha} \in \Phi^{+}$and that $b^{\prime} \in B_{\widetilde{\alpha}-1}$. Then

$$
p^{\prime} w^{\prime} b^{\prime} w_{\widetilde{\alpha}-1} \in p^{\prime} w^{\prime} b^{\prime} w_{\widetilde{\alpha}-1} B=p^{\prime} w^{\prime} w_{\widetilde{\alpha}-1} B=\left(p^{\prime} a_{-w^{\prime} \widetilde{\alpha}}\right) w^{\prime} w_{\widetilde{\alpha}} B
$$

since $w_{\widetilde{\alpha}-1} B_{\widetilde{\alpha}-1} w_{\widetilde{\alpha}-1} \subset B$. It follows that $\phi_{w, \chi}\left(p^{\prime} w^{\prime} b^{\prime} w_{\widetilde{\alpha}-1}\right)$ is equal to $\chi \delta^{1 / 2}\left(p^{\prime} a_{-w^{\prime} \widetilde{\alpha}}\right)$ if $w=w^{\prime} w_{\widetilde{\alpha}}$ and 0 otherwise.

Finally, suppose that $b^{\prime} \in B-B_{\widetilde{\alpha}-1}$. As before, it can be shown that

$$
P w^{\prime} B w_{\widetilde{\alpha}-1} B=P w^{\prime} w_{\widetilde{\alpha}} B \cup P w^{\prime} B,
$$

and furthermore that $p^{\prime} w^{\prime} b^{\prime} w_{\widetilde{\alpha}-1} \in P w^{\prime} B$ if and only if $b^{\prime}$ is an element of $B-B_{\widetilde{\alpha}-1}$. Hence $p^{\prime} w^{\prime} b^{\prime} w_{\widetilde{\alpha}-1}=p w^{\prime} b$ for some $p \in P, b \in B$. It is easily shown that this forces $p^{-1} p^{\prime} \in N P_{0}$ so that $\chi \delta^{1 / 2}(p)=\chi \delta^{1 / 2}\left(p^{\prime}\right)$. Thus $\phi_{w, \chi}\left(p^{\prime} w^{\prime} b^{\prime} w_{\widetilde{\alpha}-1}\right)$ equals $\chi \delta^{1 / 2}\left(p^{\prime}\right)$ if $w=w^{\prime}$ and 0 otherwise.

Noting that $w^{\prime} \widetilde{\alpha} \in \Phi^{ \pm}$if and only if $w^{\prime} w_{\widetilde{\alpha}} \widetilde{\alpha}=-w^{\prime} \widetilde{\alpha} \in \Phi^{\mp}$, we obtain

$$
\begin{aligned}
& \left(w_{\alpha} \cdot \phi_{w, \chi}\right)\left(p^{\prime} w^{\prime} b^{\prime}\right) \\
& \quad= \begin{cases}\chi \delta^{1 / 2}\left(a_{w \widetilde{\alpha}}\right) \chi \delta^{1 / 2}\left(p^{\prime}\right) & \text { if } w \widetilde{\alpha} \in \Phi^{-}, w^{\prime}=w w_{\widetilde{\alpha}}, b^{\prime} \in B_{\widetilde{\alpha}-1} \\
\chi \delta^{1 / 2}\left(a_{w \widetilde{\alpha}}\right) \chi \delta^{1 / 2}\left(p^{\prime}\right) & \text { if } w \widetilde{\alpha} \in \Phi^{+}, w^{\prime}=w w_{\widetilde{\alpha}} \\
\chi \delta^{1 / 2}\left(p^{\prime}\right) & \text { if } w \widetilde{\alpha} \in \Phi^{+}, w^{\prime}=w, b^{\prime} \in B-B_{\widetilde{\alpha}-1} \\
0 & \text { otherwise. }\end{cases}
\end{aligned}
$$

The second result follows.

Theorem 3.1 has the following corollary giving the action of $\operatorname{ch}_{B s B}$ for $s$ in $S_{\text {aff }}$. 
Corollary 3.2. Suppose that $w \in W, \alpha \in \Delta$ and $\widetilde{\alpha} \in \widetilde{\Delta}$. Then

$$
\begin{gathered}
\operatorname{ch}_{B w_{\alpha} B} \cdot \phi_{w, \chi}= \begin{cases}\phi_{w w_{\alpha}, \chi} & \text { if } w \alpha \in \Phi^{+} \\
q_{\alpha+1} \phi_{w w_{\alpha}, \chi}+\left(q_{\alpha+1}-1\right) \phi_{w, \chi} & \text { if } w \alpha \in \Phi^{-},\end{cases} \\
\operatorname{ch}_{B w_{\widetilde{\alpha}-1} B} \cdot \phi_{w, \chi}= \begin{cases}\chi \delta^{1 / 2}\left(a_{w \widetilde{\alpha}}\right) \phi_{w w_{\tilde{\alpha}}, \chi} & \text { if } w \widetilde{\alpha} \in \Phi^{-} \\
\chi \delta^{1 / 2}\left(a_{w \widetilde{\alpha}}\right) q_{\widetilde{\alpha}} \phi_{w w_{\tilde{\alpha}}, \chi}+\left(q_{\widetilde{\alpha}}-1\right) \phi_{w, \chi} & \text { if } w \widetilde{\alpha} \in \Phi^{+} .\end{cases}
\end{gathered}
$$

Proof. We prove the first formula in the case $w \alpha \in \Phi^{-}$. The other cases are handled similarly. For $g \in G$ we have

$$
\begin{aligned}
\left(\operatorname{ch}_{B w_{\alpha} B} \cdot \phi_{w, \chi}\right)(g) & =\int_{G} \phi_{w, \chi}(g x) \operatorname{ch}_{B w_{\alpha} B}(x) d x \\
& =\int_{B w_{\alpha} B} \phi_{w, \chi}(g x) d x \\
& =\sum_{n} \phi_{w, \chi}\left(g n w_{\alpha}\right) \\
& =\sum_{n}\left(w_{\alpha} \cdot \phi_{w, \chi}\right)(g n),
\end{aligned}
$$

where $n$ ranges over a set of representatives in $N(\alpha)$ for $N(\alpha) / N(\alpha+1)$.

If $g \in P w w_{\alpha} B$ then so is $g n$ for each of the $q_{w_{\alpha}}=q_{\alpha+1}$ representatives $n$. On the other hand, if $g \in P w B$, then $g n \in P w\left(B-B_{\alpha}\right)$ for precisely $q_{\alpha+1}-1$ of the representatives $n$. Thus

$$
\begin{aligned}
\left(\operatorname{ch}_{B w_{\alpha} B} \cdot \phi_{w, \chi}\right)(g) & =\sum_{n}\left(w_{\alpha} \cdot \phi_{w, \chi}\right)(g n) \\
& =\sum_{n}\left[\phi_{w w_{\alpha}, \chi}(g n)+\operatorname{ch}_{P w\left(B-B_{\alpha}\right)}(g n) \phi_{w, \chi}(g n)\right] \\
& =q_{\alpha+1} \phi_{w w_{\alpha}, \chi}(g)+\left(q_{\alpha+1}-1\right) \phi_{w, \chi}(g) .
\end{aligned}
$$

The following corollary of Theorem 3.1 gives a basis for $I(\chi)^{\langle B, s\rangle}, s \in S_{\text {aff }}$.

Corollary 3.3. Suppose $\alpha \in \Delta$ and $\widetilde{\alpha} \in \widetilde{\Delta}$. Then

(i) $\left\{\phi_{w, \chi}+\phi_{w w_{\alpha}, \chi} \mid w \in W, w \alpha \in \Phi^{+}\right\}$is a basis for the fixed space $I(\chi)^{\left\langle B, w_{\alpha}\right\rangle}$.

(ii) $\left\{\phi_{w, \chi}+\chi \delta^{1 / 2}\left(a_{w \widetilde{\alpha}}\right) \phi_{w w_{\tilde{\alpha}}, \chi} \mid w \in W, w \widetilde{\alpha} \in \Phi^{+}\right\}$is a basis for the fixed space $I(\chi)^{\left\langle B, w_{\tilde{\alpha}-1}\right\rangle}$.

Proof. Let $s \in S_{\text {aff. Note that }}$

$$
s \cdot I(\chi)^{B} \cap I(\chi)^{B}=I(\chi)^{s B s} \cap I(\chi)^{B}=I(\chi)^{\langle s B s, B\rangle}=I(\chi)^{\langle B, s\rangle} .
$$


Thus $I(\chi)^{\langle B, s\rangle}$ is precisely the set of vectors in $I(\chi)^{B}$ sent to $I(\chi)^{B}$ by $s$. It is clear from Theorem 3.1 that if $s=w_{\alpha}$ this set is spanned by

$$
\left\{\phi_{w, \chi}+\phi_{w w_{\alpha}, \chi} \mid w \in W, w \alpha \in \Phi^{+}\right\},
$$

and if $s=w_{\widetilde{\alpha}-1}$ this set is spanned by

$$
\left\{\phi_{w, \chi}+\chi \delta^{1 / 2}\left(a_{w \widetilde{\alpha}}\right) \phi_{w w_{\tilde{\alpha}}, \chi} \mid w \in W, w \widetilde{\alpha} \in \Phi^{+}\right\} .
$$

\section{Proof of Theorem 1.1.}

We now prove that the dimension of

$$
I(\chi)^{K}=I(\chi)^{B W_{K} B}=\bigcap_{s \in S} I(\chi)^{\langle B, s\rangle}
$$

is equal to $\left|W / \nu\left(W_{K}\right)\right|$.

Suppose that $f=\sum_{w \in W} c(w) \phi_{w, \chi}$ is a vector in $I(\chi)^{B}$ with the $c(w) \in \mathbb{C}$. Then it is easily deduced from Corollary 3.3 that $f \in \bigcap_{s \in S} I(\chi)^{\langle B, s\rangle}$ if and only if for all $w \in W$,

$$
\begin{gathered}
c\left(w w_{\alpha}\right)=c(w) \text { for all } \alpha \in \Delta \text { with } w_{\alpha} \in S \\
c\left(w w_{\widetilde{\alpha}}\right)=\chi \delta^{1 / 2}\left(a_{w \widetilde{\alpha}}\right) c(w) \text { for all } \widetilde{\alpha} \in \widetilde{\Delta} \text { with } w_{\widetilde{\alpha}-1} \in S .
\end{gathered}
$$

Let $V$ be the space of functions $c: W \rightarrow \mathbb{C}$ satisfying (3) and (4). Then $\operatorname{dim} I(\chi)^{K}=\operatorname{dim} V$. Since $\nu\left(w_{\beta-1}\right)=\nu\left(w_{\beta}\right)=w_{\beta}$ for all $\beta \in \Phi$, it follows that $c(w)$ determines $c\left(w w^{\prime}\right)$ for all $w^{\prime} \in\langle\nu(s) \mid s \in S\rangle=\nu\left(W_{K}\right)$ so

$$
\operatorname{dim} V \leq\left|W / \nu\left(W_{K}\right)\right| \text {. }
$$

We will prove that $\operatorname{dim} V=\left|W / \nu\left(W_{K}\right)\right|$.

Remark 4.1. We note that if $W_{K} \subset W$ (i.e., if $\left.K \subset K_{0}\right)$ then it is clear that $\operatorname{dim} V=\operatorname{dim} I(\chi)^{K}=\left|W / \nu\left(W_{K}\right)\right|$ since in this case only the relations in (3) appear.

Since $W_{K}$ is finite, it contains no non-trivial translations so $\nu$ is injective on $W_{K}$. Thus $\nu\left(W_{K}\right) \cong W_{K}$, and $\nu\left(W_{K}\right)$ is generated as a Coxeter group by $\nu(S)$. We will denote the element of $W_{K}$ corresponding to $t \in \nu(S)$ by $\nu^{-1}(t)$. Define recursively a function [ ] from the set of finite sequences of elements of $\nu(S)$ to $W_{\text {aff. Let }} t_{1}, \ldots, t_{n} \in \nu(S)$. For the empty sequence $\emptyset$, let $[\emptyset]=e$. Define

$$
\left[t_{1}\right]= \begin{cases}e & \text { if } \nu^{-1}\left(t_{1}\right)=w_{\alpha}, \alpha \in \Delta \\ a_{\widetilde{\alpha}} & \text { if } \nu^{-1}\left(t_{1}\right)=w_{\widetilde{\alpha}-1}, \widetilde{\alpha} \in \widetilde{\Delta}\end{cases}
$$

and then set

$$
\left[t_{1}, \ldots, t_{n}\right]= \begin{cases}{\left[t_{1}, \ldots, t_{n-1}\right]} & \text { if } \nu^{-1}\left(t_{n}\right)=w_{\alpha}, \alpha \in \Delta \\ {\left[t_{1}, \ldots, t_{n-1}\right] a_{t_{1} \cdots t_{n-1} \widetilde{\alpha}}} & \text { if } \nu^{-1}\left(t_{n}\right)=w_{\widetilde{\alpha}-1}, \widetilde{\alpha} \in \widetilde{\Delta}\end{cases}
$$


It follows easily from the definition of [ ] that

$$
\left[t_{1}, \ldots, t_{k}\right]\left(t_{1} \cdots t_{k}\right)\left[t_{k+1}, \ldots, t_{n}\right]\left(t_{1} \cdots t_{k}\right)^{-1}=\left[t_{1}, \ldots, t_{n}\right] .
$$

We claim that the element $\left[t_{1}, \ldots, t_{n}\right]$ of $W_{\text {aff }}$ depends only on the product $t_{1} \cdots t_{n}$ and not on the particular sequence $t_{1}, \ldots, t_{n}$.

Lemma 4.2. Let $t_{1}, \ldots, t_{n}, u_{1}, \ldots, u_{m}$ be elements of $\nu(S)$ such that

$$
t_{1} \cdots t_{n}=u_{1} \cdots u_{m}
$$

Then $\left[t_{1}, \ldots, t_{n}\right]=\left[u_{1}, \ldots, u_{m}\right]$.

Proof. Since $\left(\nu\left(W_{K}\right), \nu(S)\right)$ is a Coxeter group, the word $t_{1} \cdots t_{n}$ is obtainable from $u_{1} \cdots u_{m}$ via the basic Coxeter group relations among the elements of $\nu(S)$, i.e., those of the form $(t u)^{m(t, u)}=e$, where $t, u \in \nu(S)$ and $m(t, u)$ is some number in $\{1,2,3,4,6\}$ (see e.g. $[\mathbf{5}, 1.6]$ ). Therefore, it suffices to show that [ ] remains unchanged when a subsequence of consecutive terms in a sequence $t_{1}, \ldots, t_{n}$ is deleted according to such a relation. In fact, due to (5) one need only show that

$$
[\underbrace{t, u, t, u, \ldots, t, u}_{m(t, u)}]=[\emptyset]=e
$$

for each basic relation $(t u)^{m(t, u)}=e$ among the elements of $\nu(S)$.

It is clear that (6) holds if $\nu^{-1}(t), \nu^{-1}(u) \in W$. Therefore we shall consider only those relations which involve some reflection $t \in \nu(S)$ such that $\nu^{-1}(t) \notin$ $W$. Such a $t$ is necessarily of the form $w_{\widetilde{\alpha}}=\nu\left(w_{\widetilde{\alpha}-1}\right)$ for some $\widetilde{\alpha} \in \widetilde{\Delta}$. The basic relations involving $w_{\widetilde{\alpha}}$ are of the form

$$
\left(w_{\widetilde{\alpha}} u\right)^{m}=e
$$

where $u \in \nu(S)$ and $m \in\{1,2,3,4\}$. (It is never the case that $m=6$.)

First consider the case $m=1$. Here $u$ must equal $w_{\widetilde{\alpha}}$ so (6) holds as

$$
\left[w_{\widetilde{\alpha}}, w_{\widetilde{\alpha}}\right]=a_{\widetilde{\alpha}} a_{w_{\widetilde{\alpha}} \widetilde{\alpha}}=a_{\widetilde{\alpha}} a_{-\widetilde{\alpha}}=e .
$$

Now suppose that $m>1$ and $\nu^{-1}(u) \in W$ in (7). Then

$$
\underbrace{\left[w_{\widetilde{\alpha}}, u, \ldots, w_{\widetilde{\alpha}}, u\right]}_{m}=a_{\widetilde{\alpha}} \ldots a_{\left(w_{\widetilde{\alpha}} u\right)^{m-1} \widetilde{\alpha}} .
$$

Since $w_{\widetilde{\alpha}} u$ is a rotation of order $m, \widetilde{\alpha}+\cdots+\left(w_{\widetilde{\alpha}} u\right)^{m-1} \widetilde{\alpha}=0$ so (6) holds as

$$
a_{\widetilde{\alpha}} \ldots a_{\left(w_{\widetilde{\alpha}} u\right)^{m-1} \widetilde{\alpha}}=e .
$$

Finally, suppose $m>1$ and $\nu^{-1}(u) \notin W$ in (7). In this case, it follows that $m=2$ and $u=w_{\widetilde{\beta}}$ for some $\widetilde{\beta} \in \widetilde{\Delta}$. Then $w_{\widetilde{\beta}}(\widetilde{\alpha})=\widetilde{\alpha}$ and $w_{\widetilde{\alpha}}(\widetilde{\beta})=\widetilde{\beta}$. It follows that (6) holds again as

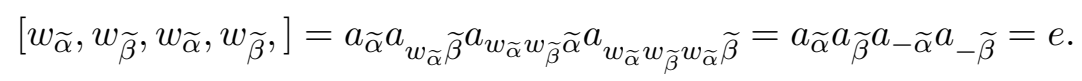


Let $t_{1}, \ldots, t_{n} \in \nu(S)$. Since $\left[t_{1}, \ldots, t_{n}\right]$ depends only on the product $t_{1} \cdots t_{n}$, [ ] gives a function $\nu\left(W_{K}\right) \rightarrow W_{\text {aff }}$, which we will also denote by [ ]. Explicitly, for $w \in \nu\left(W_{K}\right),[w]=\left[t_{1}, \ldots, t_{n}\right]$ for any $t_{1}, \ldots, t_{n} \in \nu(S)$ with $w=t_{1} \cdots t_{n}$. Note that [ ] is a 1-cocycle from $\nu\left(W_{K}\right)$ to the group of translations in $W_{\text {aff. }}$

Proposition 4.3. The space $V$ of functions $W \rightarrow \mathbb{C}$ satisfying (3) and (4) has dimension $\left|W / \nu\left(W_{K}\right)\right|$.

Proof. Let $R$ be a set of representatives for the left cosets of $\nu\left(W_{K}\right)$ in $W$. For each $\sigma \in R$, define the function $c_{\sigma}: W \rightarrow \mathbb{C}$ by setting

$$
c_{\sigma}(w)= \begin{cases}\chi \delta^{1 / 2}\left(\left[w^{\prime}\right]\right) & \text { if } w=\sigma w^{\prime} \in \sigma \nu\left(W_{K}\right) \\ 0 & \text { if } w \notin \sigma \nu\left(W_{K}\right) .\end{cases}
$$

The $c_{\sigma}$ are clearly linearly independent and are $\left|W / \nu\left(W_{K}\right)\right|$ in number. It suffices then to show that the $c_{\sigma}$ are in $V$.

Fix $\sigma \in R$. Let $\alpha$ be an element of $\Delta$ such that $w_{\alpha} \in S$. If $w \notin \sigma \nu\left(W_{K}\right)$ then $w w_{\alpha} \notin \sigma \nu\left(W_{K}\right)$ so

$$
c_{\sigma}(w)=0=c_{\sigma}\left(w w_{\alpha}\right) .
$$

If $w=\sigma w^{\prime} \in \sigma \nu\left(W_{K}\right)$ then

$$
c_{\sigma}\left(w w_{\alpha}\right)=c_{\sigma}\left(\sigma w^{\prime} w_{\alpha}\right)=\chi \delta^{1 / 2}\left(\left[w^{\prime} w_{\alpha}\right]\right)=\chi \delta^{1 / 2}\left(\left[w^{\prime}\right]\right)=c_{\sigma}(w) .
$$

Thus (3) holds for $c_{\sigma}$.

Now let $\widetilde{\alpha}$ be an element of $\widetilde{\Delta}$ such that $w_{\widetilde{\alpha}-1} \in S$. As before, if $w \notin$ $\sigma \nu\left(W_{K}\right)$ then

$$
c_{\sigma}(w)=0=\chi \delta^{1 / 2}\left(a_{w \widetilde{\alpha}}\right) c_{\sigma}\left(w w_{\widetilde{\alpha}}\right) .
$$

And if $w=\sigma w^{\prime} \in \sigma \nu\left(W_{K}\right)$ then

$$
\begin{aligned}
c_{\sigma}\left(w w_{\widetilde{\alpha}}\right) & =c_{\sigma}\left(\sigma w^{\prime} w_{\widetilde{\alpha}}\right) \\
& =\chi \delta^{1 / 2}\left(\left[w^{\prime} w_{\widetilde{\alpha}}\right]\right) \\
& =\chi \delta^{1 / 2}\left(\left[w^{\prime}\right] a_{w^{\prime} \widetilde{\alpha}}\right) \\
& =\chi \delta^{1 / 2}\left(\left[w^{\prime}\right]\right) \chi \delta^{1 / 2}\left(a_{w^{\prime} \widetilde{\alpha}}\right) \\
& =\chi \delta^{1 / 2}\left(a_{w^{\prime} \widetilde{\alpha}}\right) c_{\sigma}(w) .
\end{aligned}
$$

Thus $c_{\sigma}$ satisfies (4) and lies in $V$.

It follows that $\operatorname{dim} I(\chi)^{K}=\operatorname{dim} V=\left|W / \nu\left(W_{K}\right)\right|$. 


\section{References}

[1] I.N. Bernstein and A.V. Zelevinskii, Representations of the group $\operatorname{GL}(n, F)$, where $F$ is a non-Archimedean local field, Russian Math. Surveys, 31(3) (1976), 1-66, MR 54 \#12988, Zbl 0348.43007.

[2] P. Cartier, Representations of $\mathfrak{p}$-adic groups: A survey, Automorphic Forms, Representations, and $L$-functions (Providence, RI) (Armand Borel and William Casselman, eds.), Proc. Symp. Pure Math., 33, Amer. Math. Soc., (1977), 111-155, MR 81e:22029, Zbl 0421.22010.

[3] W. Casselman, The unramified principal series of $\mathfrak{p}$-adic groups I, Compositio Math., 40 (1980), 387-406, MR 83a:22018, Zbl 0472.22004.

[4] N. Iwahori, Generalized Tits system (Bruhat decomposition) on $\mathfrak{p}$-adic semisimple groups, Algebraic Groups and Discontinuous Subgroups (Providence, RI) (Armand Borel and George D. Mostow, eds.), Proc. Symp. Pure Math., 9, Amer. Math. Soc., (1966), 71-89, MR 35 \#6693, Zbl 0199.06901.

[5] N. Iwahori and H. Matsumoto, On some Bruhat decompositions and the structure of the Hecke ring of $\mathfrak{p}$-adic Chevalley groups, Inst. Hautes Études Sci. Publ. Math., 25 (1965), 237-280, MR 32 \#2486.

[6] J. Tits, Reductive groups over local fields, Automorphic Forms, Representations, and $L$-functions (Providence, RI) (Armand Borel and William Casselman, eds.), Proc. Symp. Pure Math., 33, Amer. Math. Soc., (1977), 29-69, MR 80h:20064, Zbl 0415.20035.

Received August 1, 2000 and revised January 9, 2001.

Department of Mathematics

UNIVERSITY OF ROCHESTER

Rochester, New YORK 14627

E-mail address: lansky@math.rochester.edu 